\title{
Anti-CD22 epratuzumab for systemic lupus erythematosus: A systematic review and meta-analysis of randomized controlled trials
}

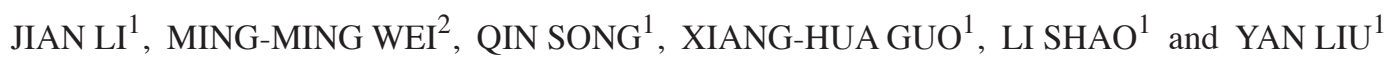 \\ Departments of ${ }^{1}$ Rheumatology and Immunology and ${ }^{2}$ Nephrology, Affiliated Hospital \\ of Jining Medical University, Jining, Shandong 272000, P.R. China
}

Received August 31, 2017; Accepted January 23, 2018

DOI: $10.3892 /$ etm.2019.7630

\begin{abstract}
Systemic lupus erythematosus (SLE) is a remarkable and challenging autoimmune disorder that is characterized by a broad range of clinical manifestations, such as flares and remissions. Recently, the humanized anti-CD22 antibody epratuzumab for SLE has been extensively studied. The aim of the present study was to perform a meta-analysis on the findings of associated randomized controlled trials in order to evaluate the effects of epratuzumab on SLE. Data from publications in PubMed, EMBASE and the Cochrane Library were collected up to March 2017. To calculate the risk ratio or standardized mean differences (SMDs) with 95\% confidence intervals (CIs), a random effect model was applied when heterogeneity was significant and a fixed effect model was used when heterogeneity was negligible. All statistical tests were performed using Review Manager 5.3 software. A total of 1,921 participants in 4 studies (5 trials) that met the selection criteria were analyzed in this meta-analysis. Analyses of the BILAG-based Combined Lupus Assessment (BICLA) response and SLE Disease Activity Index 2000 (SLEDA-2K) score revealed that epratuzumab (720-3,600 mg) significantly improved the BICLA response $(\mathrm{RR}=1.09$; 95\% CI, 1.04 to 1.14) and decreased the SLEDA-2K score (SMD=-0.31; 95\% CI, -0.67 to $0.06 ; \mathrm{P}=0.10$ ). While the British Isles Lupus Assessment Group index score was not significantly altered between the epratuzumab and control groups. For safety analyses, no statistically significant differences were identified between the two groups, which were proved by the pooled results (all P-values $>0.05$ ). These findings suggested that epratuzumab may be relatively safe and may have better
\end{abstract}

Correspondence to: Professor Ming-Ming Wei, Department of Nephrology, Affiliated Hospital of Jining Medical University, 89 Guhuai Road, Jining, Shandong 272000, P.R. China

E-mail: chase_dream123@163.com

Key words: systemic lupus erythematosus, epratuzumab, efficacy, safety, meta-analysis therapeutic effectiveness than placebo control conditions in patients with SLE.

\section{Introduction}

Systemic lupus erythematosus (SLE) is a chronic, archetypal, relapsing-remitting, multisystem autoimmune inflammatory disease, with an estimated incidence of 5-50 cases per 100,000 people (1). This disease affects somewhere between 200,000 and 500,000 people in the USA and more than 1000,000 individuals in China, predominantly young women in their reproductive years (2). It is encouraging that the five-year survival rate for SLE has already surpassed $80 \%(3,4)$. However, the mortality still cannot be ignored, with nearly $10 \%$ risk of death within 10 years of diagnosis (5). Various severe clinical manifestations severely affect the life quality. Especially, up to $80 \%$ of SLE patients are suffering from various different mucocutaneous symptoms, such as butterfly rash, oral ulcers, chronic cutaneous lupus erythematosus, and increased photosensitivity (6).

Over the past decade, targeted therapies for SLE have lagged behind other autoimmune rheumatic disorders due to the varying degrees in multiple organ systems (7). But the good news is a range of biologic agents have shown their potential clinical effects in the management of SLE (8). Since SLE is characterized by polyclonal B cell hyper-reactivity that is considered to be the most important pathogenic event (9). Recently, anti-B cell antibody therapies have been widely investigated for the treatment of SLE, and have exhibited encouraging results regarding their efficacy and safety in clinical trials. Those reported anti-B cell agents mainly include anti-CD20 antibodies (rituximab, ofatumumab, and ocrelizumab), anti-CD22 antibody (epratuzumab), and anti-BAFF-R/BCMA/TACI receptors agents (belimumab and atacicept) (10). Among them, the effects of rituximab and belimumab in SLE patients have been discussed in several meta-analyses $(8,11,12)$. On the other hand, emerging studies, especially randomized controlled trials (RCTs), emphasized the efficacy of epratuzumab for the management of SLE.

As a member of the Siglec protein family, the CD22 membrane receptor is a B-lymphocyte-restricted adhesion molecule that plays a role in B-cell activation and interaction 
with T cells (13). The loss of CD22 function could contribute to the pathogenesis of autoimmune diseases, including SLE (14). Epratuzumab is a humanized monoclonal antibody, which can rapidly induce a marked decrease of CD22 (>80\%) and slight inhibition of other proteins from the B-cell surface in vitro, including CD19, CD21 and CD $79 \beta(15,16)$. Initially, epratuzumab was used as a promising immunotherapy for non-Hodgkin's lymphoma (13). But a few years later, a phase I trial reported that the total BILAG (British Isles Lupus Activity Group) score was decreased in $92 \%$ SLE participants after 18-week epratuzumab treatment (17). After that, more trials with larger samples and longer follow-up were performed to evaluate the possibilities for improvement in SLE patients. Wallace et al found that treatment with epratuzumab was well tolerated in patients with moderately to severely active SLE, and associated with improvements in disease activity $(18,19)$. Tsuru and colleagues also demonstrated the inhibitory effects of epratuzumab on CD22 and B cell count in SLE patients (20). However, Clowse et al (21) argued that epratuzumab did not result in improvements for SLE patients.

Although these results are variable and controversial, up to now, no quantitatively systematic review has been conducted in this field. Therefore, meta-analysis is urgently required to summarize the available evidence to assess the potential clinical effects of epratuzumab in the management of SLE (22). The main aim of this meta-analysis is to synthesize findings from published RCTs assessing the efficacy and safety in SLE patients.

\section{Materials and methods}

Search strategy. We attempted to search the published RCTs that investigated the effects of epratuzumab for the treatment of SLE patients. The relevant studies were identified and selected by searching the electronic databases of PubMed, Embase, and the Cochrane Library (updated to March 2017). The following terms were searched: 'lupus', 'systemic lupus', 'systemic lupus erythematosus', 'SLE', 'epratuzumab', 'anti-CD22 antibody', 'anti-CD22', 'CD22 targeted' and 'risk.' Both the subject words, such as Medical Subject Heading (MeSH) or Emtree terms, and random words were used to retrieve the trials comprehensively. Terms were moderately expanded within each electronic database whenever necessary. There was no language restriction in the literature search. With regard to published studies with overlapping data by the same authors, we selected the most recent or complete study only.

Inclusion and exclusion. Studies fulfilling the following inclusion criteria were eligible for this meta-analysis: i) Evaluation of the epratuzumab treatment for SLE; ii) reported the efficacy or safety of epratuzumab; and iii) RCTs. The exclusion criteria of our meta-analysis included i) studies unrelated to SLE; ii) review, case report, conference abstract, or any other non-RCTs; iii) interventions without epratuzumab; iv) duplicative or overlapping publications; and v) trials with fewer than 10 patients. The inclusion and exclusion criteria were confirmed according to the results of searching.

Data extraction. The whole process of data extraction was completed by a single investigator (J.L.) to assure uniformity of data and then re-evaluated by a second investigator (M.-M.W.). Any disagreement was resolved by consensus. The following data from articles that met criteria were abstracted: i) First author's name; ii) publish year; iii) sample size; iv) age of the participants; v) percent of women participants; vi) patient characteristics; vii) interventions (dose and methods of administration); viii) control condition; ix) follow-up duration (endpoint); and x) outcome measures for efficacy and safety. It should be noted that we utilized the data for meta-analysis at the highest dose of epratuzumab (720-3,600 mg) and the longest endpoint (12 or 48 weeks).

Assessment of quality. We independently graded the strength of evidence for each outcome in accordance with the Cochrane criteria (23) (J.L. and Q.S.). This quality assessment system was composed of the adequacy of sequence generation, allocation concealment, blinding, addressing of dropouts (incomplete outcome data), selective outcome reporting, and other potential sources of bias. An assessment of 'yes' indicated a low risk of bias, while 'no' indicated a high risk of bias. Labeling an item as 'unclear' indicated an unclear or unknown risk of bias (23). Ethical approval or additional consent from participants was not provided since this study was a systematic review of published studies. Ethical approval or additional consent from patients was not provided because the present study was a literature review of previously reported studies.

Statistical analysis. All data analysis was performed with the Review Manager 5.3 software from the Cochrane Collaboration. Unadjusted risk ratios (RRs) with a 95\% confidence interval (CI) were calculated for binary outcomes. For continuous variables, standardized mean differences (SMDs) and $95 \%$ CIs were calculated by measuring the post-treatment difference between the mean of the epratuzumab treatment and the mean of control condition, divided by the pooled standard deviation (SD). When necessary, the mean and SD were estimated from the median, range, and the size of a sample according to Hozo's formulas (24). The effect of heterogeneity between studies rather than sampling error was quantified by using an $\mathrm{I}^{2}$ statistic as well as a P-value. If $\mathrm{I}^{2} \geq 50 \%$ or P-value $\leq 0.1$, a random effect model was adopted. Otherwise, a fixed effect model was used. Funnel plots were not performed to evaluate publication bias due to the limited number of included studies. P-value $\leq 0.5$ was considered as the significance level.

\section{Results}

Study selection. The process of selection is shown in a PRISMA flow diagram (Fig. 1). Our search returned 773 publications and abstracts, of which 108 were identified as duplicates using Endnote X7 software and excluded. A total of 600 studies were removed based on title or abstract review. The remaining publications were further identified by reading the full text. After careful assessment, four articles (five trials) met the inclusion criteria and were selected for meta-analysis (18-21). Specifically, three studies resulted eligible to evaluate effectiveness $(18,19,21)$, and four resulted eligible to evaluate safety (18-21).

Study characteristics. The characteristics of the included trials are summarized in Table I. In brief, all studies were published from 2013 to 2017. A total of 1,921 subjects were included in the four eligible RCTs, comprising the epratuzumab group 


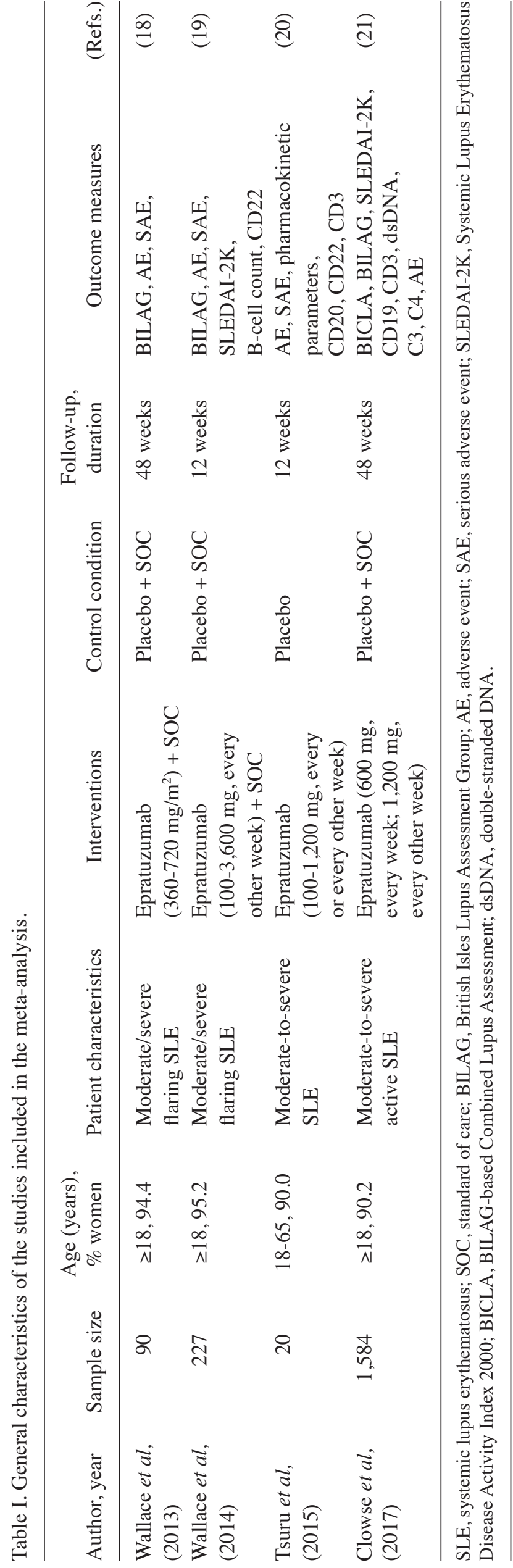

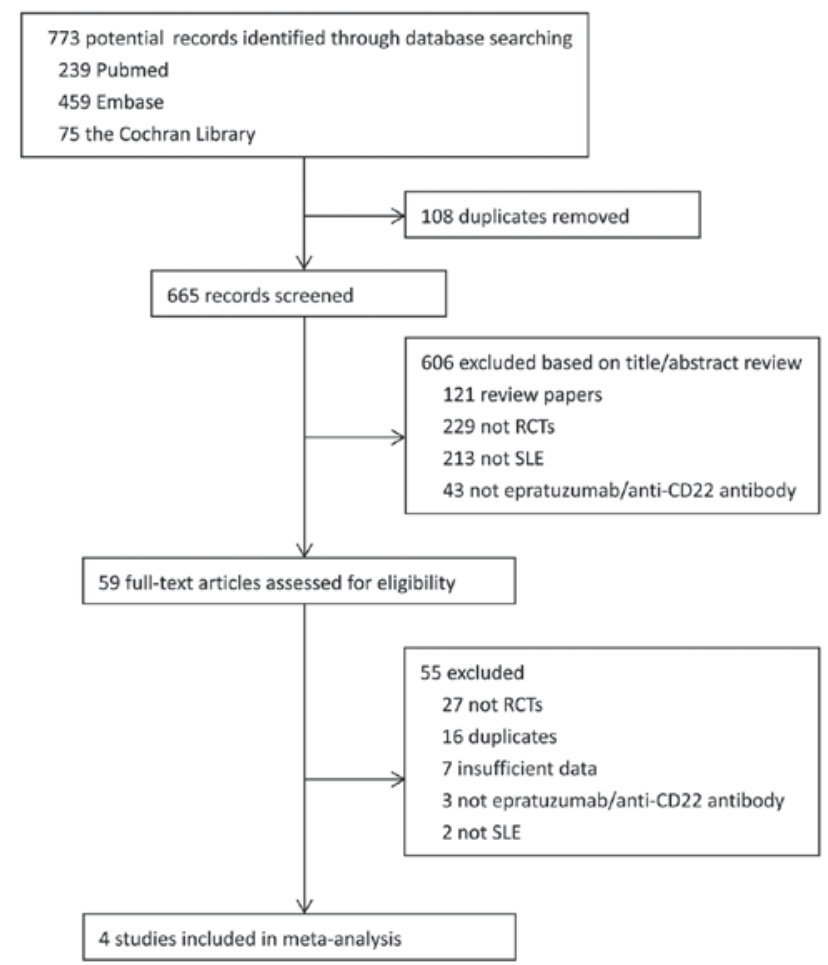

Figure 1. Preferred reporting items for systematic reviews and meta-analyses flow chart of the study selection process used in the meta-analysis. SLE, systemic lupus erythematosus; RCTs, randomized controlled trials; CD22, cluster of differentiation 22 .

and the control group. The largest study had a population size of 1,584 subjects (21), while the smallest study recruited 20 subjects (20). It is worth mentioning that two RCTs (EMBODY 1 and EMBODY 2), 793 patients and 791 patients, respectively, were included in the largest study (21). Therefore, EMBODY 1 and EMBODY 2 were analyzed respectively in the meta-analysis. All studies recruited moderate-to-severe SLE participants aged $\geq 18$ years. The percentage of female patients is more than $90 \%$. A wide range of dosages of epratuzumab (100-3,600 mg every week or every other week) was used in the included trials. Only the highest dosage in the original trial was utilized for analysis. The control conditions were placebo (20) or placebo + standard of care (SOC) $(18,19,21)$. Duration of studies ranged between 12 weeks $(19,20)$ and 48 weeks $(18,21)$. All studies were designed as randomized, double-blind, placebo-controlled trials.

Study quality. Overall, three trials ( $75 \%$ ) had adequate sequence generation and adequate concealed allocation (19-21). All selected studies used blinding and reported the complete and unselective outcomes. Nevertheless, other potential sources of bias were sufficiently addressed by some included trials. Although randomization has been conducted before grouping, the baseline condition of one trial was not very consistent (19). One study had a high drop-out rate $(>30 \%)$, which may be resulted from the long follow-up duration (21). Table II presents the summary details on the risk of bias among included trials.

Efficacy of epratuzumab for SLE. Since not every included trial reported follow-up effects and there were also differences in the follow-up time, the present meta-analysis aimed to evaluate the 
Table II. Risk of bias assessment in the studies considered for meta-analysis.

\begin{tabular}{lcccccc}
\hline Author, year & $\begin{array}{c}\text { Random sequence } \\
\text { generation }\end{array}$ & $\begin{array}{c}\text { Allocation } \\
\text { concealment }\end{array}$ & Blinding & $\begin{array}{c}\text { Incomplete } \\
\text { outcome data }\end{array}$ & $\begin{array}{c}\text { Selective } \\
\text { reporting }\end{array}$ & $\begin{array}{c}\text { Free of } \\
\text { other bias }\end{array}$ \\
Wallace et al, 2013 & $\mathrm{U}$ & $\mathrm{U}$ & $\mathrm{L}$ & $\mathrm{L}$ & $\mathrm{L}$ & $\mathrm{L}$ \\
(Refs.)
\end{tabular}

Criteria defined for quality assessment was based on the Cochrane guidelines. H, high risk of bias; L, low risk of bias; U, unclear or unrevealed risk of bias.

\begin{tabular}{|c|c|c|c|c|c|c|}
\hline & Epratuzı & mab & Contrc & & & Risk Ratio \\
\hline Author, Year & Events & Total & Events & Total & Weight & $\mathrm{M}-\mathrm{H}$, Fixed, $95 \% \mathrm{Cl}$ \\
\hline Wallace et al, 2013 & 17 & 44 & 9 & 30 & $1.7 \%$ & $1.29[0.66,2.50]$ \\
\hline Wallace et al, 2014 & 63 & 190 & 8 & 38 & $2.2 \%$ & $1.57[0.82,3.01]$ \\
\hline Clowse et al, 2017-1 & 468 & 523 & 212 & 263 & $46.0 \%$ & $1.11[1.04,1.19]$ \\
\hline Clowse et al, 2017-2 & 478 & 525 & 231 & 263 & $50.1 \%$ & $1.04[0.98,1.09]$ \\
\hline Total $(95 \% \mathrm{Cl})$ & & 1282 & & 594 & $100.0 \%$ & $1.09[1.04,1.14]$ \\
\hline Total events & 1026 & & 460 & & & \\
\hline Heterogeneity: $\mathrm{Chi}^{2}=$ & $01, \mathrm{df}=3$ & $(P=0.1$ & 17); $I^{2}=40$ & & & \\
\hline Test for overall effect: & $=3.59(\mathrm{P}$ & $=0.000$ & & & & \\
\hline
\end{tabular}
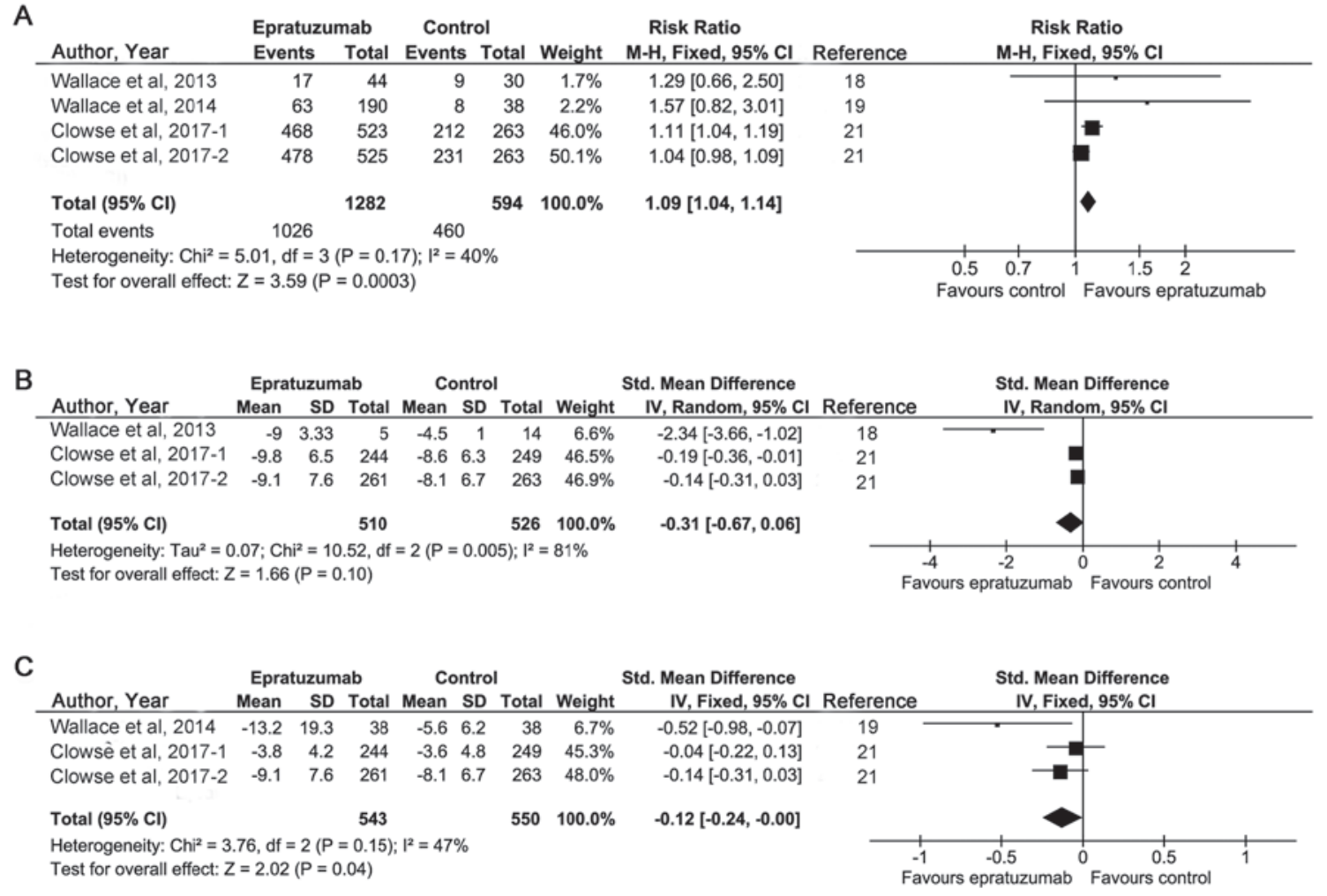

Figure 2. Meta-analysis of the studies evaluating the efficacy of epratuzumab. (A) BILAG-based Combined Lupus Assessment response, (B) BILAG index score and (C) Systemic Lupus Erythematosus Disease Activity Index 2000 score. SD, standard deviation; M-H, Mantel-Haenszel; BILAG, British Isles Lupus Assessment Group; CI, confidence intervals.

immediate post-treatment effects of epratuzumab. Some tools can be used as the efficacy variables for SLE, such as British Isles Lupus Assessment Group index (BILAG), the BILAG-based Combined Lupus Assessment (BICLA), SLE Disease Activity Index 2000 (SLEDAI-2K), Systemic Lupus International Collaborating Clinics American College of Rheumatology (SLICC ACR), and Safety of Estrogens in Systemic Lupus Erythematosus National Assessment-Systemic Lupus Erythematosus Disease Activity Index (SELENA-SLEDAI). Any measured parameters for disease activity were pooled in the meta-analysis if they were conducted not less than 2 trials. Therefore, BICLA response, BILAG score and SLEDA-2K score were analyzed in the review.

The results showed that there were not significant heterogeneities in BICLA response and SLEDA-2K score, as was evident from $I^{2}<50 \%$. Thus, fixed effect models were adopted in these analyses. However, in the analysis of BILAG score, a significant heterogeneity was in existence and a random effect model was used. As shown in Fig. 2A, pooling trials that reported BICLA response got an RR of 1.09 (95\% CI, 1.04 to 1.14), which was a significant effect in favor of epratuzumab $(\mathrm{P}=0.0003)$. However, the mean effect size for BILAG score was slightly but not markedly in favor of epratuzumab $(\mathrm{SMD}=-0.31 ; 95 \% \mathrm{CI},-0.67$ to $0.06 ; \mathrm{P}=0.10$ ) (Fig. 2B). The results of SLEDA-2K score indicated that epratuzumab could significantly improve SLE disease activity (SMD=-0.12; 95\% CI, -0.24 to $0.00 ; \mathrm{P}=0.04$ ) (Fig. 2C). These findings show that epratuzumab has greater therapeutic effectiveness than placebo control condition in SLE patients. Subgroup meta-analyses of various ethnicities, treatment durations, and drug concentrations were not performed due to the limited amount of RCTs. 


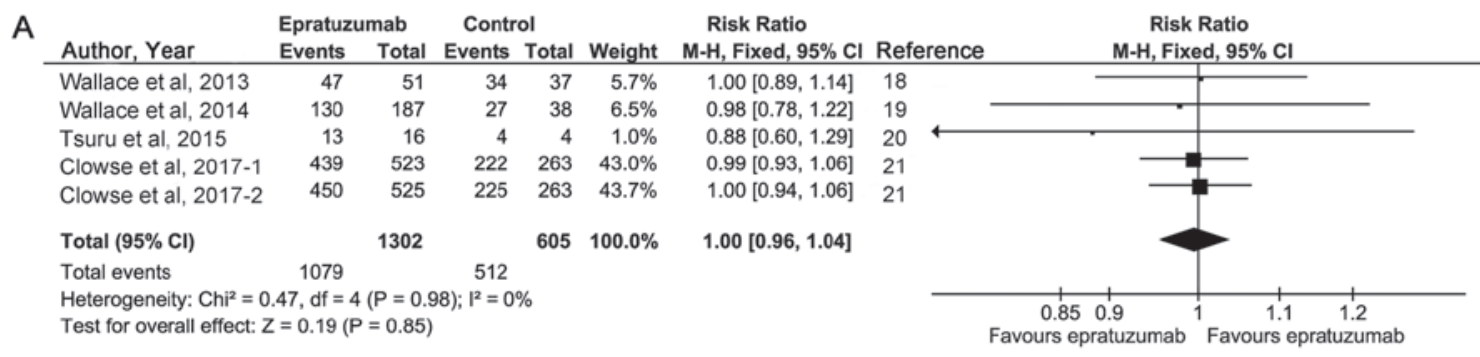

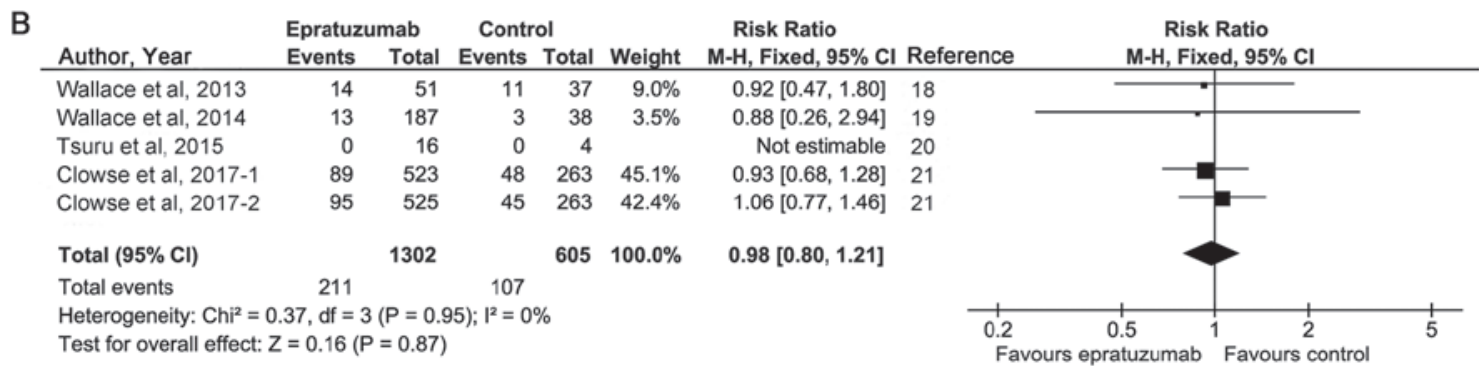

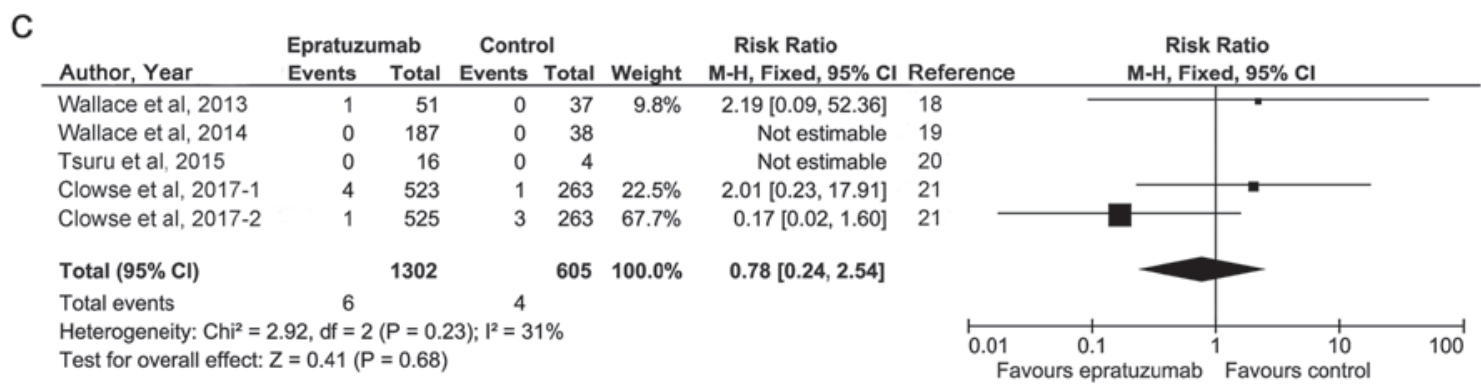

Figure 3. Meta-analysis of the studies evaluating the safety of epratuzumab. (A) Adverse events, (B) serious adverse events and (C) mortalities. M-H, Mantel-Haenszel; CI, confidence intervals.

Safety of epratuzumab for SLE. The safety of epratuzumab therapy on SLE disease activity was reported in four randomized controlled studies (five trials). Here, we conducted the meta-analyses of adverse event (AE), serious adverse event (SAE) and death to reflect the safety of epratuzumab treatment. The most common AEs in patients included headache, nausea, upper respiratory tract infection, and so on. An absence of heterogeneity among the studies included in these analyses was observed $\left(\mathrm{I}^{2}<50 \%\right)$. Therefore, fixed effect models were used in the safety analyses. Statistical analyses revealed the absence of statistically significant differences between the epratuzumab group and control group, indicating the excellent safety profile of this drug for SLE treatment. In detail, the pooled results were $(\mathrm{RR}=1.00 ; 95 \% \mathrm{CI}, 0.96$ to $1.04 ; \mathrm{P}=0.85)$ for $\mathrm{AE},(\mathrm{RR}=0.98 ; 95 \% \mathrm{CI}, 0.80$ to $1.21 ; \mathrm{P}=0.87)$ for $\mathrm{SAE}$, and $(\mathrm{RR}=0.78 ; 95 \% \mathrm{CI}, 0.24$ to $2.54 ; \mathrm{P}=0.68)$ for death. These results are presented in Fig. $3 \mathrm{~A}-\mathrm{C}$, respectively.

\section{Discussion}

SLE is a multiorgan autoimmune disease influenced by various interdependent cellular pathways and complex genetics $(25,26)$. B-lineage cells can induce specific immune responses whereby their antigen-presenting effect, and respond to antigenic challenge as effector cells of the humoral immune response. Therefore, they are regarded as the pivotal regulator involved in the pathogenesis of SLE (27). Besides anti-CD20 therapy, increasing studies have focused on the safety and activity of humanized anti-CD22 antibody epratuzumab for SLE since the initial clinical trial conducted by Dörner et al in 2006 (17).

Epratuzumab can evoke CD22 phosphorylation and restrictedly bind to B-lymphomas and B-lymphocytes, consistent with the expression of CD22 $(28,29)$. In a rodent model, epratuzumab takes a pivotal role in regulating the signaling transduction pathways in B cells, while the number and functions of B cells do not change significantly (30). It has been proved that the epratuzumab takes its immunosuppressive action via inhibiting the production of some proinflammatory cytokines in B cells, such as IL-6 and TNF- $\alpha$ (31). In the past few years, several systematic reviews have evaluated the potential clinic effects of epratuzumab as a biologic therapy in SLE, and shown the great promise with safe profiles and quality of life (32-34). However, a recent phase III trial demonstrated that epratuzumab was not effective in SLE (35). For the included RCTs, some RCTs reported the positive effects of epratuzumab in SLE patients $(18,19)$, while Clowse and the colleagues argued that epratuzumab treatment with SOC did not lead to improvements in response rates over that observed in the placebo treatment with SOC (21). 
Since there are some controversially reported data about epratuzumab therapy in SLE patients, the aim of the present work was to comprehensively determine the efficacy and safety of epratuzumab in patients with SLE. Using an exhaustive search strategy, we identified four high-quality clinic studies (five RCTs) to evaluate the intervention of epratuzumab for SLE. This meta-analysis demonstrates that epratuzumab may contribute to the improvements in SLE disease activity. Specifically, compared to control groups, epratuzumab significantly increased the BICLA response and reduced the SLEDA-2K score. Although BILAG score did not reach to statistically significant change in epratuzumab group, the BILAG score did decrease markedly compared with control group (SMD=-0.31; $95 \% \mathrm{CI},-0.67$ to $0.06 ; \mathrm{P}=0.10)$. This result may be affected by the large heterogeneity, which can hardly be explored due to the limited trials. On the other hand, epratuzumab is relatively safe compared with control group. The incidence of AEs, SAEs, and deaths was similar between epratuzumab and control group (all P-values $>0.05$ ), and no additional safety signals were found in the original studies after epratuzumab treatment. Aaccording to Wallace et al (19), treatment with epratuzumab 2,400 mg cumulative dose was well tolerated in SLE patients, and associated with improvements in disease activity.

It should also be noted that the dosage and administration frequency of epratuzumab are quite variable in different included trials, ranging from 100 to $3,600 \mathrm{mg}$ and once to twice a week. In the present meta-analysis, we chose the highest dosage to summarize the effects of epratuzumab. If we focus on Fig. 2, we can find that the treatment outcomes in the studies of Wallace et al (19) are much better than other studies. After analyzing the study design among these trials, we find that infusion of $600 \mathrm{mg}$ epratuzumab once a week for four consecutive weeks (cumulative dose 2,400 mg) may be an acceptable therapeutic strategy of epratuzumab for SLE patients.

To ensure the high quality, the present meta-analysis only identified RCTs. As shown in Table I, all included RCTs were published in recent five years (2013-2017). Since women were more susceptible to SLE than men $(36,37)$, the included female patients accounted for more than $90 \%$. The inclusion criteria and exclusion criteria of these RCTs were similar, although a great diversity of diagnostic methods were used, including ACR, BILAG index, SLEDAI-2K, SLEDAI. All participants were more than 18 years old and suffering from moderate-to-severe SLE. Most studies had low drop-out rates except one (21). All the RCTs were phase I/II blinding trials, recruiting various populations including Caucasian, Black, Asian, Hispanic/Latino and Mixed race. We can also found that the heterogeneities among various data are so small as to be ignorable in most comparisons except BILAG score $\left(\mathrm{I}^{2}=81 \%, \mathrm{P}=0.005\right)$. Therefore, take together, the included studies are of high quality, and the findings of meta-analysis are reliable, valid, and robust.

Although the results of this study suggest some promising clinical benefits of epratuzumab therapy for SLE, some limitations that may reduce our ability to draw conclusions from these findings should also be acknowledged. First of all, different studies reported different outcomes to reflect the efficacy of epratuzumab, which make them difficult to blend in the meta-analysis. Especially, SLE-associated laboratory parameters (such as $\mathrm{C} 3, \mathrm{C} 4$ levels, and dsDNA) (38) cannot be used to conduct a meta-analysis. Further studies in this field should take the same measurement criteria to detect the efficacy and safety for SLE, such as SLEDAI-2K and BILAG-2004. Besides, the number of published RCTs included in our meta-regression analysis would affect the results of this study. The relatively small samples limit the ability of the Egger's test and funnel plot to show publication bias and may lead to false positive and negative conclusions (39). Furthermore, most participants were divided in either epratuzumab + SOC group or placebo + SOC group. Nevertheless, one trial did not clearly state the control condition (20). The different condition of the control group may also affect our conclusion. Finally, the different dosages of epratuzumab (100-3,600 mg) and endpoints of treatment (12-48 weeks) may also affect the meta-analysis results.

Taken together, our meta-analysis demonstrates that anti-CD22 antibody epratuzumab may be an effective option for the treatment of moderately-to-severely active SLE. And epratuzumab appears to have an acceptable safety profile in these patients. Further RCTs with large sample size and long follow-up duration are needed to verify the effects of epratuzumab. We also expect that the comparisons between epratuzumab and other anti-B cell therapies can be focused to select the better one. It is also important to explore the efficacy of combination therapy, which has been shown to be effective in non-Hodgkin's lymphoma (40).

\section{Acknowledgements}

Not applicable.

\section{Funding}

No funding was received.

\section{Availability of data and materials}

The datasets used and/or analyzed during the current study are available from the corresponding author on reasonable request.

\section{Authors' contributions}

JL and MMW contributed to the study design, data extraction, quality assessment, analysis and interpretation of data, and drafting of the manuscript. QS contributed to the study design, quality assessment and analysis, interpretation of data and revising the article. XHG contributed to the study design, data extraction, and analysis and interpretation of data. LS and YL contributed to the conception of the study, study design and revising the article. All authors proofread and approved the submitted version of the article.

\section{Ethics approval and consent to participate}

This study was conducted based on published data. No ethics approval and consent to participate was required for this study.

\section{Consent for publication}

Not applicable 


\section{Competing interests}

The authors declare that they have no competing interests.

\section{References}

1. Uramoto KM, Michet CJ Jr, Thumboo J, Sunku J, O'Fallon WM and Gabriel SE: Trends in the incidence and mortality of systemic lupus erythematosus, 1950-1992. Arthritis Rheum 42: 46-50, 1999.

2. Lim SS, Bayakly AR, Helmick CG, Gordon C, Easley KA and Drenkard C: The incidence and prevalence of systemic lupus erythematosus, 2002-2004: The Georgia Lupus Registry. Arthritis Rheumatol 66: 357-368, 2014.

3. Lin H, Wei JC, Tan CY, Liu YY, Li YH, Li FX, Deng DH, Yan B, Liu Y and Zhao Y: Survival analysis of late-onset systemic lupus erythematosus: A cohort study in China. Clin Rheumatol 31: $1683-1689,2012$

4. Tan JH, Hoh SF, Win MT, Chan YH, Das L and Arkachaisri T: Childhood-onset systemic lupus erythematosus in Singapore: Clinical phenotypes, disease activity, damage, and autoantibody profiles. Lupus 24: 998-1005, 2015.

5. Cervera R, Khamashta MA, Font J, Sebastiani GD, Gil A, Lavilla P, Mejía JC, Aydintug AO, Chwalinska-Sadowska H, de Ramón E, et al: Morbidity and mortality in systemic lupus erythematosus during a 10-year period: A comparison of early and late manifestations in a cohort of 1,000 patients. Medicine (Baltimore) 82: 299-308, 2003.

6. Stockinger T, Richter L, Kanzler M, Melichart-Kotik M, Pas H, Derfler K, Schmidt E and Rappersberger K: Systemic lupus erythematosus: Unusual cutaneous manifestations. Hautarzt 67: 970-981, 2016 (In German).

7. Wallace DJ: The evolution of drug discovery in systemic lupus erythematosus. Nat Rev Rheumatol 11: 616-620, 2015.

8. Borba HH, Wiens A, de Souza TT, Correr CJ and Pontarolo R: Efficacy and safety of biologic therapies for systemic lupus erythematosus treatment: Systematic review and meta-analysis. BioDrugs 28: 211-228, 2014.

9. Jovancevic B, Lindholm C and Pullerits R: Anti B-cell therapy against refractory thrombocytopenia in SLE and MCTD patients: Long-term follow-up and review of the literature. Lupus 22 : 664-674, 2013

10. Faurschou M and Jayne DR: Anti-B cell antibody therapies for inflammatory rheumatic diseases. Annu Rev Med 65: 263-278, 2014.

11. Wei LQ, Liang YG, Zhao Y, Liang HT, Qin DC and She MC: Efficacy and safety of belimumab plus standard therapy in patients with systemic lupus erythematosus: A meta-analysis. Clin Ther 38 1134-1140, 2016

12. Lan L, Han F and Chen JH: Efficacy and safety of rituximab therapy for systemic lupus erythematosus: A systematic review and meta-analysis. J Zhejiang Univ Sci B 13: 731-744, 2012.

13. Leonard JP and Link BK: Immunotherapy of non-Hodgkin's lymphoma with hLL2 (epratuzumab, an anti-CD22 monoclonal antibody) and Hu1D10 (apolizumab). Semin Oncol 29: 81-86, 2002.

14. Dörner T, Shock A and Smith KG: CD22 and autoimmune disease. Int Rev Immunol 31: 363-378, 2012.

15. Rossi EA, Goldenberg DM, Michel R, Rossi DL, Wallace DJ and Chang CH: Trogocytosis of multiple B-cell surface markers by CD22 targeting with epratuzumab. Blood 122: 3020-3029, 2013.

16. Dorner T, Shock A, Goldenberg DM and Lipsky PE: The mechanistic impact of CD22 engagement with epratuzumab on B cell function: Implications for the treatment of systemic lupus erythematosus. Autoimmun Rev 14: 1079-1086, 2015.

17. Dörner T, Kaufmann J, Wegener WA, Teoh N, Goldenberg DM and Burmester GR: Initial clinical trial of epratuzumab (humanized anti-CD22 antibody) for immunotherapy of systemic lupus erythematosus. Arthritis Res Ther 8: R74, 2006.

18. Wallace DJ, Gordon C, Strand V, Hobbs K, Petri M, Kalunian K, Houssiau F, Tak PP, Isenberg DA, Kelley L, et al: Efficacy and safety of epratuzumab in patients with moderate/severe flaring systemic lupus erythematosus: Results from two randomized, double-blind, placebo-controlled, multicentre studies (ALLEVIATE) and follow-up. Rheumatology (Oxford) 52: 1313-1322, 2013.

19. Wallace DJ, Kalunian K, Petri MA, Strand V, Houssiau FA, Pike M Kilgallen B, Bongardt S, Barry A, Kelley L and Gordon C: Efficacy and safety of epratuzumab in patients with moderate/severe active systemic lupus erythematosus: Results from EMBLEM, a phase IIb, randomised, double-blind, placebo-controlled, multicentre study. Ann Rheum Dis 73: 183-190, 2014.
20. Tsuru T, Tanaka Y,Kishimoto M, Saito K, Yoshizawa S, Takasaki Y, Miyamura T, Niiro H, Morimoto S, Yamamoto J, et al: Safety, pharmacokinetics, and pharmacodynamics of epratuzumab in Japanese patients with moderate-to-severe systemic lupus erythematosus: Results from a phase 1/2 randomized study. Mod Rheumatol 26 87-93, 2016.

21. Clowse ME, Wallace DJ, Furie RA, Petri MA, Pike MC, Leszczyński P, Neuwelt CM, Hobbs K, Keiserman M, Duca L, et al: Efficacy and safety of epratuzumab in moderately to severely active systemic lupus erythematosus: Results from two phase III randomized, double-blind, placebo-controlled trials. Arthritis Rheumatol 69: 362-375, 2017.

22. Mao S and Huang S: Association between vitamin D receptor gene BsmI, FokI, ApaI and TaqI polymorphisms and the risk of systemic lupus erythematosus: A meta-analysis. Rheumatol Int 34: 381-388, 2014.

23. Sahebkar A, Rathouska J, Derosa G, Maffioli P and Nachtigal P: Statin impact on disease activity and C-reactive protein concentrations in systemic lupus erythematosus patients: A systematic review and meta-analysis of controlled trials. Autoimmun Rev 15: 344-353, 2016.

24. Hozo SP, Djulbegovic B and Hozo I: Estimating the mean and variance from the median, range, and the size of a sample. BMC Med Res Methodol 5: 13,2005.

25. Morawski PA and Bolland S: Expanding the B cell-centric view of systemic lupus erythematosus. Trends Immunol 38: 373-382, 2017.

26. Teruel M and Alarcón-Riquelme ME: The genetic basis of systemic lupus erythematosus: What are the risk factors and what have we learned. J Autoimmun 74: 161-175, 2016.

27. Dörner T and Lipsky PE: Beyond pan-B-cell-directed therapy-new avenues and insights into the pathogenesis of SLE. Nat Rev Rheumatol 12: 645-657, 2016.

28. Wallace DJ and Goldenberg DM: Epratuzumab for systemic lupus erythematosus. Lupus 22: 400-405, 2013.

29. Carnahan J, Wang P, Kendall R, Chen C, Hu S, Boone T, Juan T, Talvenheimo J, Montestruque S, Sun J, et al: Epratuzumab, a humanized monoclonal antibody targeting CD22: Characterization of in vitro properties. Clin Cancer Res 9: 3982S-3990S, 2003.

30. Özgör L, Brandl C, Shock A and Nitschke L: Epratuzumab modulates B-cell signaling without affecting B-cell numbers or B-cell functions in a mouse model with humanized CD22. Eur J Immunol 46: 2260-2272, 2016.

31. Fleischer V, Sieber J, Fleischer SJ, Shock A, Heine G, Daridon C and Dörner T: Epratuzumab inhibits the production of the proinflammatory cytokines IL- 6 and TNF- $\alpha$, but not the regulatory cytokine IL-10, by B cells from healthy donors and SLE patients. Arthritis Res Ther 17: 185, 2015.

32. Rao V and Gordon C: Evaluation of epratuzumab as a biologic therapy in systemic lupus erythematosus. Immunotherapy 6 : 1165-1175, 2014.

33. Al Rayes $\mathrm{H}$ and Touma Z: Profile of epratuzumab and its potential in the treatment of systemic lupus erythematosus. Drug Des Devel Ther 8: 2303-2310, 2014

34. Antoniu S: Epratuzumab for systemic lupus erythematosus. Expert Opin Biol Ther 14: 1045-1047, 2014.

35. Onuora S: Systemic lupus erythematosus: Epratuzumab not effective in phase III trials. Nat Rev Rheumatol 12: 622, 2016.

36. Achour A, Mankaï A, Thabet Y, Sakly W, Braham F, Kechrid C, Bahri $F$, Bouajina $E$, Chouchène $S$, Haddad $O$ and Ghedira I: Systemic lupus erythematosus in the elderly. Rheumatol Int 32: 1225-1229, 2012.

37. Rahman A and Isenberg DA: Systemic lupus erythematosus. N Engl J Med 358: 929-939, 2008

38. Lim KL, Jones AC, Brown NS and Powell RJ: Urine neopterin as a parameter of disease activity in patients with systemic lupus erythematosus: Comparisons with serum sIL-2R and antibodies to dsDNA, erythrocyte sedimentation rate, and plasma C3, C4 and C3 degradation products. Ann Rheum Dis 52: 429-435, 1993.

39. Gong H, Ni CX, Liu YZ, Zhang Y, Su WJ, Lian YJ, Peng W and Jiang CL: Mindfulness meditation for insomnia: A meta-analysis of randomized controlled trials. J Psychosom Res 89: 1-6, 2016.

40. Strauss SJ, Morschhauser F, Rech J, Repp R, Solal-Celigny P, Zinzani PL, Engert A, Coiffier B, Hoelzer DF, Wegener WA, et al: Multicenter phase II trial of immunotherapy with the humanized anti-CD22 antibody, epratuzumab, in combination with rituximab, in refractory or recurrent non-Hodgkin's lymphoma. J Clin Oncol 24: 3880-3886, 2006. 